\title{
MIRA: Joint regulatory modeling of multimodal expression and chromatin accessibility in single cells
}

\author{
Allen W. Lynch* 1,2, Christina V. Theodoris ${ }^{* 1,3-4}$, Henry Long ${ }^{2,5}$, Myles Brown ${ }^{2,5}$, X. Shirley \\ Liü 1-2, 6 , Clifford A. Meyer\# 1-2, 6 \\ ${ }^{1}$ Department of Data Science, Dana-Farber Cancer Institute, Boston MA, USA. \\ ${ }^{2}$ Center for Functional Cancer Epigenetics, Dana-Farber Cancer Institute, Boston, MA, USA. \\ ${ }^{3}$ Division of Genetics and Genomics, Boston Children's Hospital, Boston MA, USA. \\ ${ }^{4}$ Harvard Medical School Genetics Training Program, Boston, USA. \\ ${ }^{5}$ Department of Medical Oncology, Dana-Farber Cancer Institute, Brigham and Women's \\ Hospital, and Harvard Medical School, Boston, MA, USA. \\ ${ }^{6}$ Department of Biostatistics, Harvard T.H. Chan School of Public Health, Boston, MA, USA. \\ * Contributed equally. \\ \# Co-corresponding authors.
}

Correspondence to: cliff_meyer@ds.dfci.harvard.edu, xsliu.res@gmail.com

Rigorously comparing gene expression and chromatin accessibility in the same single cells could illuminate the logic of how coupling or decoupling of these mechanisms regulates fate commitment. Here, we present MIRA: Probabilistic Multimodal Models for Integrated Regulatory Analysis, a comprehensive methodology that systematically contrasts transcription and accessibility to infer the regulatory circuitry driving cells along developmental trajectories. MIRA leverages joint topic modeling of cell states and regulatory potential modeling of individual gene loci. MIRA thereby represents cell states in an efficient and interpretable latent space, infers high fidelity lineage trees, determines key regulators of fate decisions at branch points, and exposes the variable influence of local accessibility on transcription at distinct loci. Applied to epidermal maintenance differentiation and embryonic brain development from two different multimodal platforms, MIRA revealed that early developmental genes were tightly regulated by local chromatin landscape whereas terminal fate genes were titrated without requiring extensive chromatin remodeling.

Main

Profiling both expression and chromatin accessibility in the same single cells $\mathrm{s}^{1-5}$ opens an unprecedented opportunity to understand the interaction of transcriptional and epigenetic mechanisms driving cells along developmental continuums. While many computational methods analyze expression and accessibility separately, several recent algorithms have adopted joint analysis where the cells are projected onto a shared latent space based on both data modalities, which better captures the biological structure of the data ${ }^{6-11}$. However, the field lacks tools that go beyond visualization and clustering to rigorously contrast transcription and 
accessibility in each single cell to illuminate the complex regulatory circuitry driving developmental fate decisions.

Integrated analysis of global transcriptional and accessibility states across developmental trajectories would enable discovery of key regulators controlling fate decisions at lineage branch points. At the gene level, examining the dynamics of transcription versus chromatin accessibility proximal to the gene locus may reveal how these mechanisms interact to regulate distinct gene modules. Certain genes may be regulated by cis-regulatory elements that are simultaneously activated as they become accessible, whereas others may be regulated by elements whose accessibility and activation are decoupled ${ }^{12,13}$. Determining the logic of which genes are regulated by each of these distinct mechanisms may provide insight into the patterns of pathways that demand tight spatiotemporal regulation versus signal responsivity.

Here, we present MIRA: Probabilistic Multimodal Models for Integrated Regulatory Analysis, a comprehensive methodology that systematically contrasts transcription and accessibility to determine the regulatory circuitry driving cells along developmental continuums. MIRA leverages joint topic modeling of cell states and regulatory potential (RP) modeling of individual gene loci. MIRA thereby represents cell states in an efficient and interpretable latent space, infers high fidelity lineage trees, determines key regulators of fate decisions at branch points, and exposes the variable influence of local accessibility on transcription at distinct loci. We applied MIRA to an epidermal maintenance differentiation ${ }^{3}$ and brain developmental system $^{14}$ assayed by multimodal single cell RNA-sequencing (scRNA-seq) and Assay for Transposase-Accessible Chromatin-sequencing (scATAC-seq) data from two different platforms (SHARE-seq and 10x). In each system, MIRA constructed a high fidelity developmental trajectory and determined the regulatory factors driving key fate decisions at lineage branch points. Furthermore, MIRA distinguished early developmental genes that were tightly spatiotemporally regulated by local chromatin landscape from terminal fate genes that were permitted to remain accessible while titrated by factors with minimal impact on local chromatin, revealing how variable regulatory circuitry coordinates fate commitment and terminal identity.

\section{Results}

\section{MIRA leverages joint topic modeling and RP modeling to reveal the circuitry regulating developmental trajectories}

MIRA leverages joint topic modeling and RP modeling of expression and chromatin accessibility in single cells to determine the regulatory mechanisms driving key fate decisions within developmental continuums (Fig. 1a-b, Methods). Probabilistic topic modeling has been employed in natural language understanding to elucidate the abstract topics that shape the meaning of a given collection of text ${ }^{15}$. Recently, topic modeling has been applied to scRNA-seq and ScATAC-seq separately to describe either transcriptional or epigenetic cell states ${ }^{16,17}$ as "thematic" groups of co-regulated genes or cis-regulatory elements, respectively.

MIRA's topic model uses a variational autoencoder ${ }^{18}$ approach, intersecting deep learning with probabilistic graphical models, to learn expression and accessibility topics defining each cell's identity (Extended Data Fig. 1). MIRA accounts for the distinct statistical properties of each modality by using different generative distributions for overdispersed scRNA-seq counts and sparse scATAC-seq data. A sparsity constraint is employed to ensure cells' topic compositions are coherent and interpretable ${ }^{19}$. MIRA's Bayesian hyperparameter tuning scheme 
finds the appropriate number of topics needed to comprehensively yet non-redundantly describe each dataset.

MIRA next combines the expression and accessibility topics into a joint representation used to calculate a k-nearest neighbors (KNN) graph. The KNN graph is then leveraged to construct a high fidelity lineage tree using a new method we developed to define the branch points between lineages where the probabilities of differentiating into one terminal state diverges from another (Extended Data Fig. 2). MIRA then contrasts the emergence of expression and accessibility topics mapped on this lineage tree to elucidate the key regulators driving fate decisions at the inferred lineage branch points.

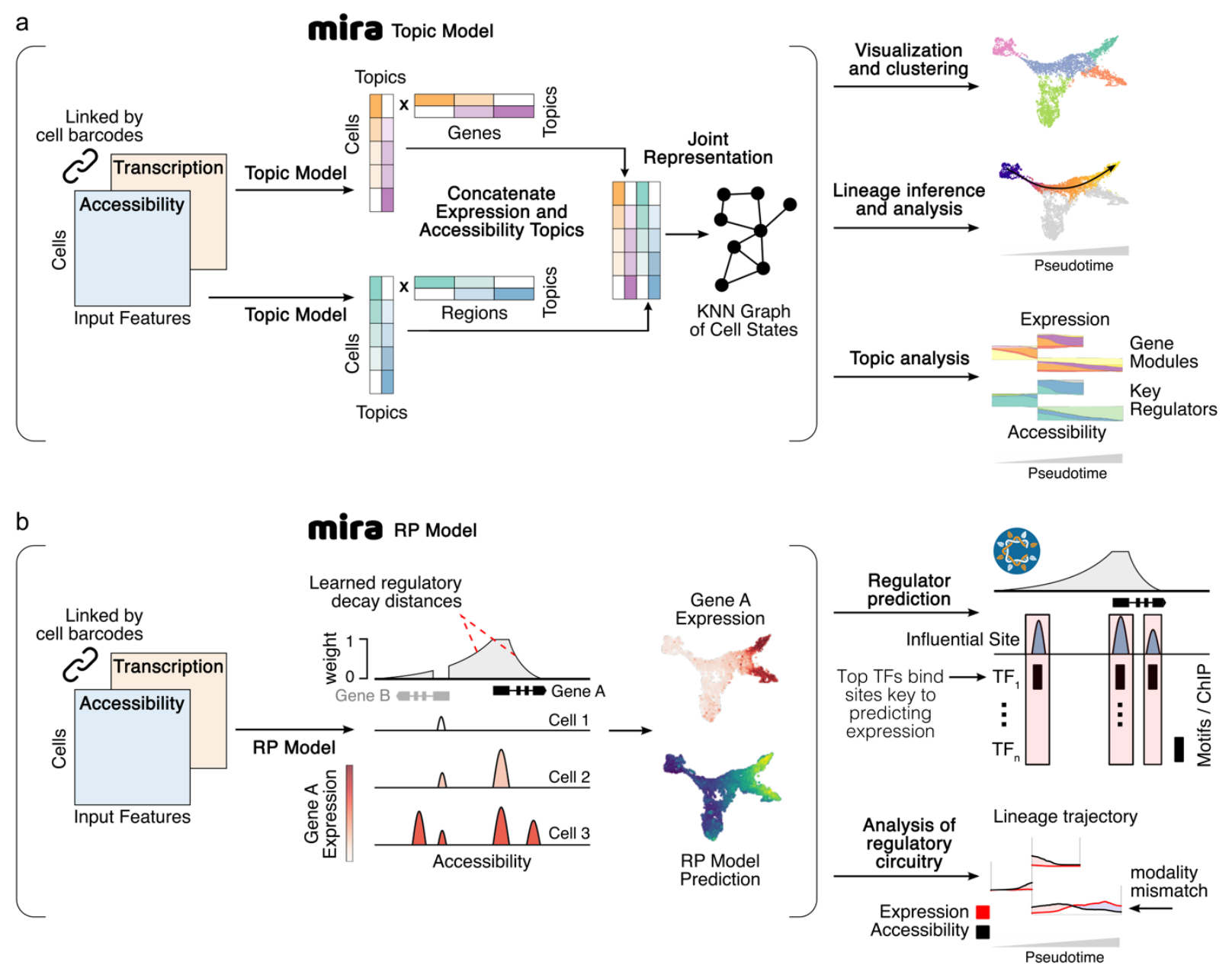

Fig. 1 | Schematic of MIRA's cell-level topic and gene-level RP models for deeply integrated analysis of single cell multimodal transcription and accessibility data. a, MIRA harnesses a variational autoencoder ${ }^{18}$ approach to model the transcription and chromatin accessibility topics defining each cell's identity with a sparsity constraint to ensure topics are coherent and interpretable ${ }^{19}$. MIRA's hyperparameter tuning scheme learns the appropriate number of topics needed to comprehensively yet non-redundantly describe each dataset. MIRA next combines the expression and accessibility topics into a joint representation used to calculate a k-nearest neighbors (KNN) graph. This output can then be leveraged for visualization and clustering, construction of high fidelity lineage trajectories, and rigorous topic analysis to determine regulators driving key fate decisions at lineage branch points. b, MIRA's RP model integrates transcriptional and chromatin accessibility data at each gene locus to determine how regulatory elements surrounding each gene influence its expression. Regulatory influence of enhancers is modeled to decay exponentially with genomic distance at a rate learned by the MIRA RP model from the joint multimodal data. MIRA learns 
independent upstream and downstream decay rates and includes parameters to weigh upstream, downstream, and promoter effects. MIRA quantifies the regulatory influence of local chromatin state by contrasting the true gene expression to that which would be predicted based solely on the local accessibility state (LITE model) or an expanded model that also includes the genome-wide accessibility state encoded by MIRA accessibility topics (NITE model). This quantification enables MIRA to distinguish genes primarily regulated by local chromatin remodeling (LITE genes) versus those more heavily influenced by non-local signals (NITE genes) reflected in the genome-wide accessibility topics with minimal impact on local chromatin landscape. MIRA furthermore predicts key regulators at each locus by examining transcription factor motif or occupancy (from ChIP-seq) enrichment within elements predicted to highly influence transcription at that locus via a pISD approach.

Next, MIRA leverages RP modeling ${ }^{20,21}$ to integrate transcription and accessibility at the resolution of individual gene loci to determine how regulatory elements surrounding each gene influence its expression (Fig. 1b). While correlation between chromatin accessibility and expression is confounded by coordinated genome-wide changes ascribed to cell state, genomic proximity suggests a mechanistic regulatory relationship between cis-regulatory elements and transcription. Thus, the perceived influence of cis-regulatory elements is modeled to decay exponentially with genomic distance upstream or downstream of a transcriptional start site (TSS) at independent rates learned by MIRA from the multimodal data. Each gene's RP is scored as the sum of the contribution of individual regulatory elements. MIRA predicts key regulators at each locus by examining transcription factor motif enrichment or occupancy (if provided chromatin immunoprecipitation-sequencing (ChIP-seq) data) within elements predicted to highly influence transcription at that locus by probabilistic in silico deletion (pISD).

Furthermore, MIRA quantifies the regulatory influence of local chromatin accessibility on gene expression by comparing the local RP model with a second, expanded model augmented with knowledge of genome-wide accessibility states encoded by MIRA's accessibility topics. Genes whose transcription is sufficiently predicted by the RP model based on local accessibility alone ( \pm 600 kilobases from the TSS) are defined as local chromatin accessibility-influenced transcriptional expression (LITE) genes. Genes whose expression is significantly better described by the model with genome-wide scope are defined as non-local chromatin accessibility-influenced transcriptional expression (NITE) genes.

While LITE genes appear tightly regulated by local chromatin accessibility, the transcription of NITE genes appears to be titrated without requiring extensive local chromatin remodeling. MIRA defines the extent to which the LITE model over- or under-estimates expression in each cell as "chromatin differential", highlighting cells where transcription is decoupled from shifts in local chromatin accessibility. MIRA examines chromatin differential across the developmental continuum to reveal how variable circuitry regulates fate commitment and terminal identity.

\section{MIRA joint topic modeling determined regulators driving key fate decisions in hair follicle differentiation}

Applied to hair follicle maintenance differentiation assayed by SHARE-seq ${ }^{3}$, MIRA's joint topic representation constructed a lineage map whose latent structure mimicked the follicle's true spatial layout ${ }^{22,23}$ (Fig. 2a, Extended Data Fig. 3). MIRA's inferred lineage tree reconstructed the ancestral hierarchy of follicular lineages, with outer root sheath cells leading to early matrix progenitors, which subsequently branched into descendant inner root sheath (IRS) followed by 
medulla and cortex lineages (Fig. 2b). Accurate lineage trees are a crucial prerequisite to determining the factors directing cell fate decisions at lineage branch points.

a

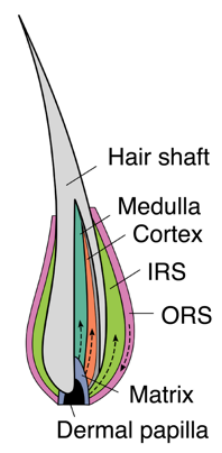

C
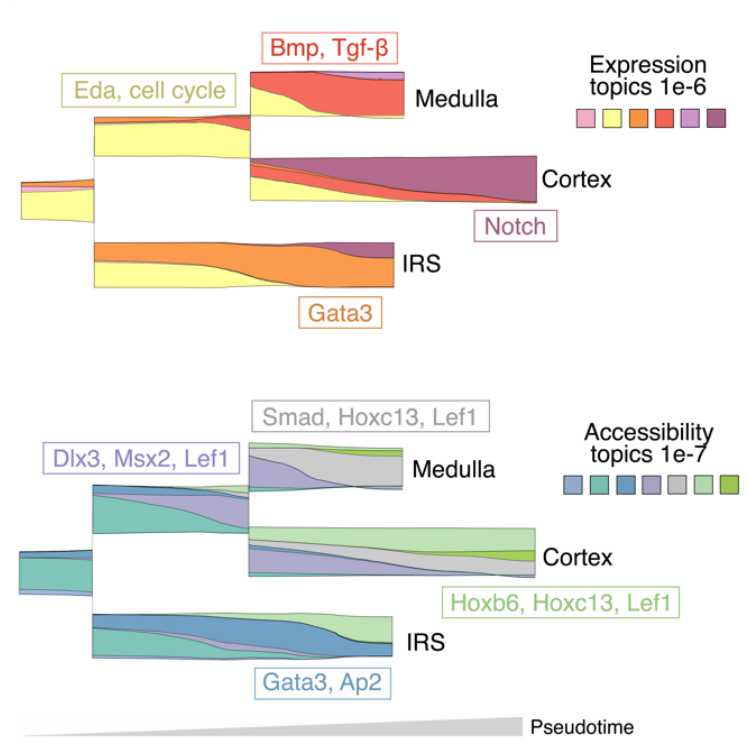

b

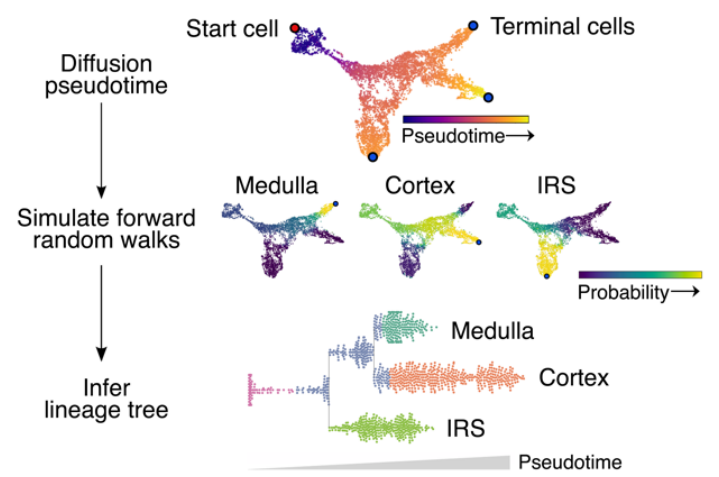

d

Expression topic e2: WikiPathways enrichments
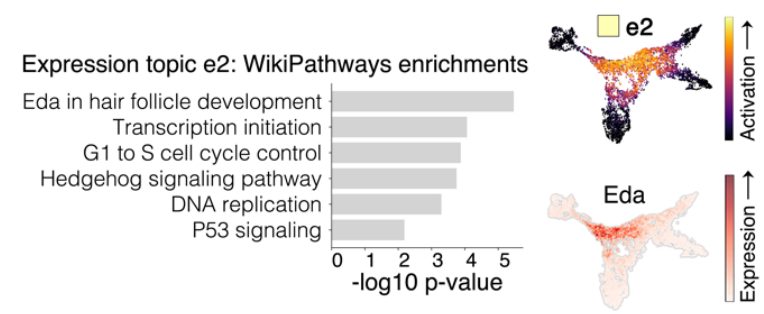

e

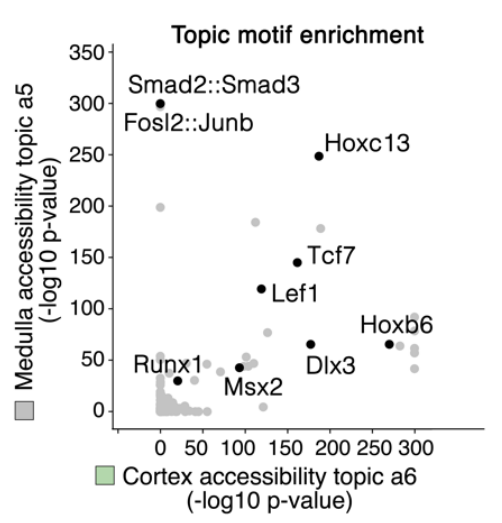

Fig. 2 | MIRA joint topic modeling determined regulatory factors driving key fate decisions in hair follicle differentiation. a, MIRA's joint topic representation constructed a UMAP (right) whose structure mimicked the true spatial layout ${ }^{22}$ (left) of the progenitor matrix cells and descendant medulla, cortex, and IRS lineages in the hair follicle. b, (top) Diffusion pseudotime through joint KNN graph representing differentiation progress. Terminal cells were identified using stationary states from a forward Markov chain model of differentiation. (middle) Each cell's probability of reaching each terminal state. (bottom) Parsed bifurcating tree structure of lineage probabilities visualized as stream graph. Cells colored by clusters in 2a. c, Stream graph of window-averaged cell-topic compositions as cells progress through differentiation starting from matrix cell state (see Extended Data Fig. 4a for stream graph including outer root sheath (ORS)). Representative genes activated in expression topics and motifs enriched in accessibility topics are depicted in boxes corresponding with the color of the source topic. Accessibility topic a4 described a transitory accessibility state at the branch point between the medulla and cortex lineages without any corresponding expression topic, suggesting global chromatin remodeling in progenitor matrix cells preceded transcriptional alterations specifying each downstream lineage. d, (left) Gene set enrichment for progenitor matrix cell expression topic e2. (right) Expression topic e2 activation or Eda expression on UMAP of joint topic representation. e, Comparison of motif enrichment in top peaks of medulla versus cortex accessibility topics (a5 and a6, respectively).

MIRA contrasts the flow of expression and accessibility topics across the inferred lineage tree using stream graphs ${ }^{24}$ to expose the regulatory modules driving cell fates along 
distinct developmental paths (Fig. 2c; Extended Data Fig. 4a-b, Table 1-2). Stream graphs enable high-dimensional, multimodal comparisons along continuums. Expression topic e2 captured the transcriptional state governing progenitor matrix cells, including cell proliferation ${ }^{25}$ and Eda and Shh signaling ${ }^{22,26}$ (Fig. 2d). Thereafter, expression topic e6 described cortex specification corresponding with activation of Notch-associated factors ${ }^{27}$. Conversely, expression topic e4 characterized medulla specification, containing Bmp/Tgf- $\beta$-associated factors $^{22}$ aligned with enrichment of Smad5/Smad2/3 motifs in medulla-specific accessibility topic a5 (Fig. 2e, Extended Data Fig. 4c). Comparison with cortex-specific accessibility topic a6 showed both lineages were enriched for motifs bound by canonical hair shaft regulators Lef1 and $\mathrm{Hoxc}^{22}$, with expression implicating the influence of Hoxc13 (Extended Data Fig. 5a).

Contrasting modalities, Wnt-driven accessibility topic a4 described a transitory accessibility state at the branch point between the medulla and cortex lineages without any corresponding expression topic (Fig. 2c, Extended Data Fig. 5b). Cell-level chromatin remodeling in progenitor matrix cells thus preceded transcriptional alterations specifying each downstream lineage.

\section{MIRA RP modeling distinguished LITE versus NITE genes in the hair follicle}

While most genes in the hair follicle exhibited LITE regulation with local accessibility increasing synchronously with transcription, expression diverged from that predicted by the LITE model for genes such as Krt23 (Fig. 3a-b, Extended Data Fig. 5c). Although local chromatin accessibility was poorly predictive of Krt23 expression, its transcription was lineage-specific and closely aligned with activation of accessibility topic a5, encoding a medulla genome-wide pattern of accessibility. Consistently, Krt23 expression was more closely predicted by the NITE model which includes these genome-wide accessibility states as features (Fig. 3c). LITE genes are thus tightly regulated by local chromatin remodeling, whereas NITE genes are titrated without requiring extensive local chromatin remodeling, decoupling transcription from local accessibility (Fig. 3d).

MIRA's "chromatin differential" mapped the extent to which local accessibility was decoupled from transcription across the developmental trajectory (Fig. 3e-f). Although Krt23 local accessibility increased at the branch point between the medulla and cortex lineages and remained elevated in both, it was ultimately only highly expressed in the medulla, causing high chromatin differential that over-estimated its expression in the cortex. Krt23's lineage-specific expression despite accessibility in both lineages suggests its activation requires addition of a factor that does not primarily impact transcription via remodeling local accessibility.

\section{MIRA analysis of NITE regulation elucidated hair follicle fate commitment mechanism} At the cell level, gene expression in terminally-differentiated medulla and cortex cells exhibited significantly more NITE regulation than gene expression earlier in hair follicle differentiation ( $p<0.05$, Wilcoxon) (Fig. 4a, Extended Data Fig. 5d). Often, accessibility of terminally-expressed genes increased before fate commitment and was maintained in both subsequent lineages, but expression activated in a lineage-specific manner only after the branch point between medulla and cortex (Fig. 4b-c, Extended Data Fig. 5e). We used chromatin differential at the branch point to identify genes with these "branch-primed" dynamics. While priming suggests the 
inevitability of expression, these genes indicate subsequent expression at primed loci can be conditional, a pattern detected as strong NITE regulation.

a

Aligned accessibility and expression: Braf

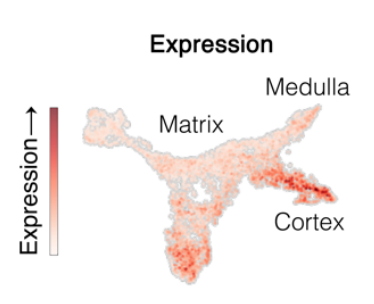

b

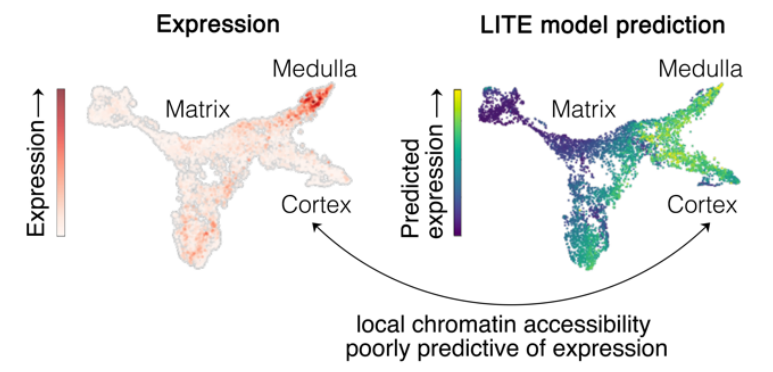

C
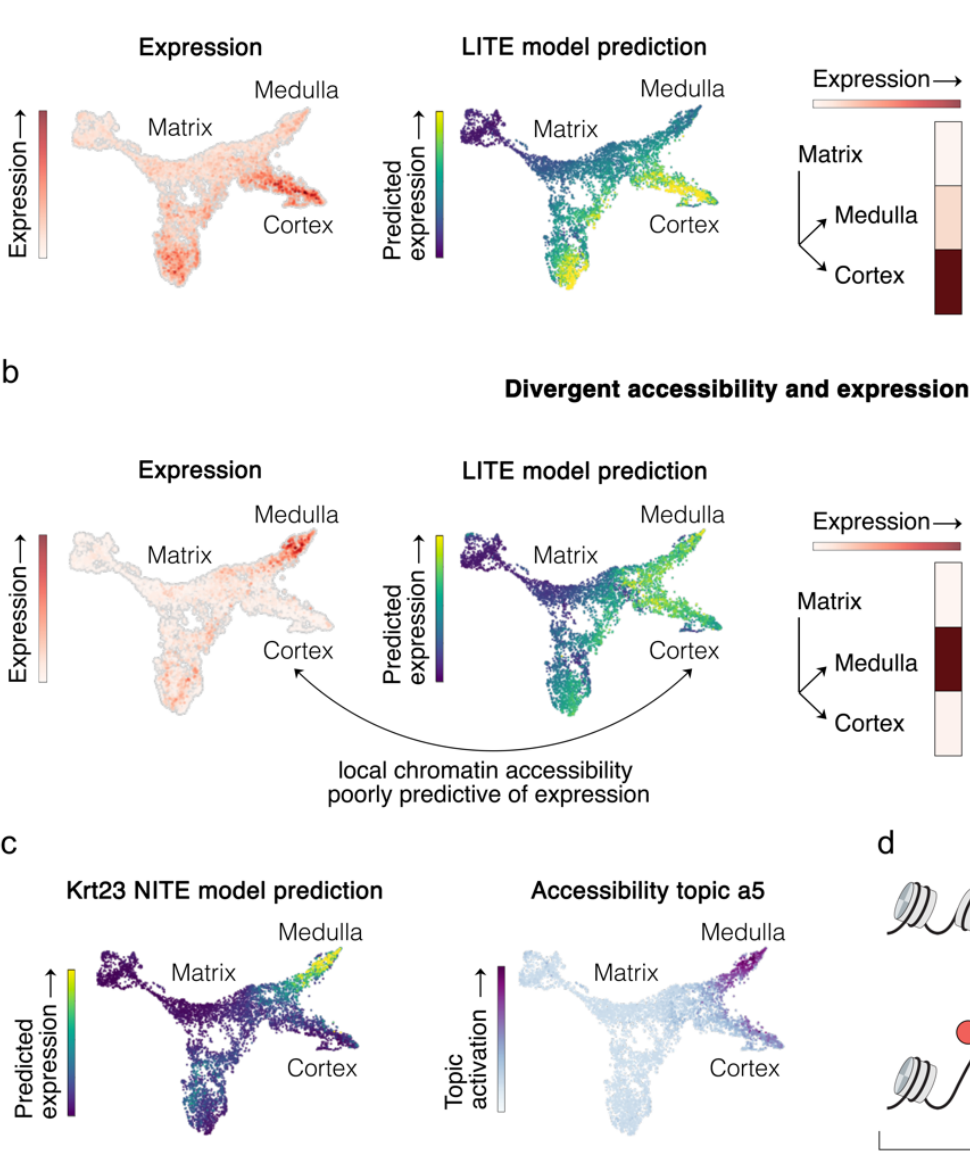

$$
\underset{\leftarrow}{50 \mathrm{~kb}} \longmapsto \leftarrow \leftarrow \leftarrow 4 \text { Braf }
$$

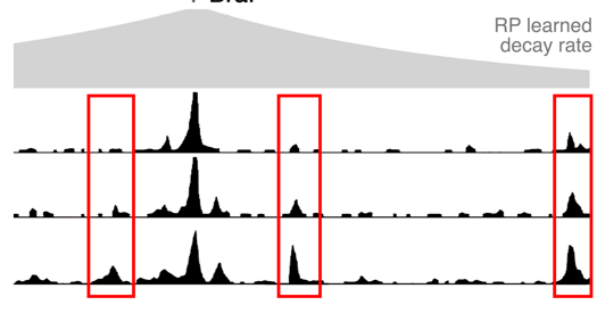

Divergent accessibility and expression: Krt23

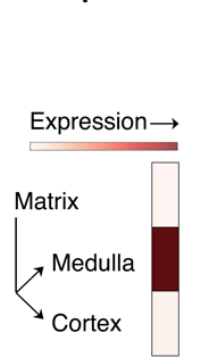

$50 \mathrm{~kb}$

.......... Krt23 Krt39

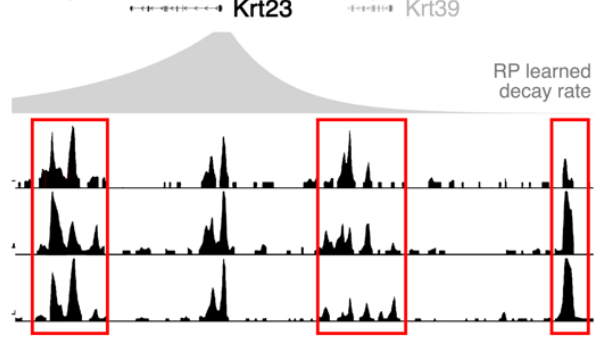

d

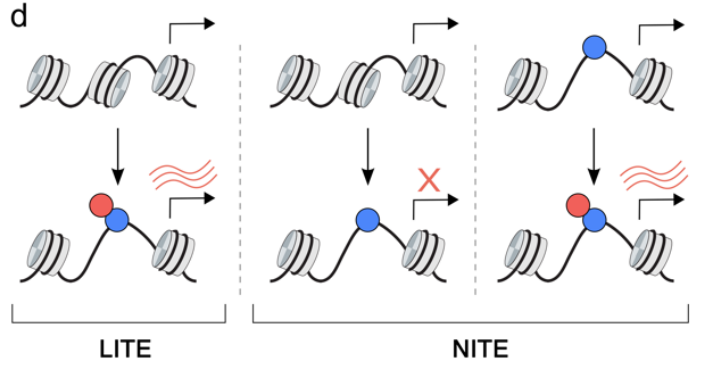

e

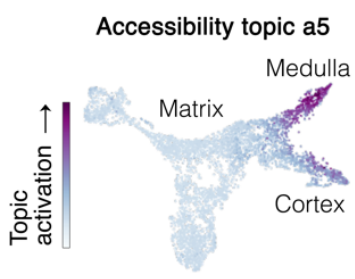

LITE gene: Braf
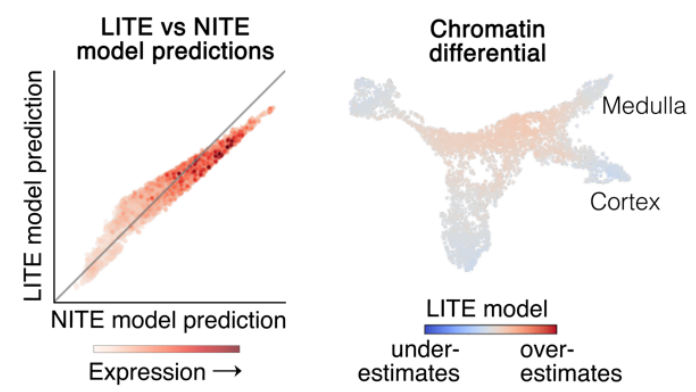

Fig. 3 | MIRA RP modeling identified genes for which changes in expression were insufficiently explained by local chromatin accessibility. a, LITE gene Braf or b, NITE gene Krt23 expression or local-only RP model predictions (LITE model) on joint representation UMAP (left) and (right) chromatin accessibility tracks as cells progressed from matrix branch into medulla or cortex states, indicating primarily LITE versus NITE regulation, respectively. c, Joint representation UMAP colored by (left) Krt23 NITE model prediction or (right) medulla accessibility topic a5 capturing a genome-wide chromatin state. NITE model predictions were more closely aligned with Krt23 expression shown in 3b. d, Proposed mechanism of LITE versus NITE regulation. In LITE regulation, expression is tightly regulated by chromatin remodeling. In NITE regulation, binding of an additional factor is required 
to enact transcription. e, LITE gene Braf or $\mathbf{f}$, NITE gene Krt23 LITE versus NITE model predictions (cells colored by gene expression) and "chromatin differential" (relative prediction of LITE versus NITE models). In chromatin differential plots, red indicates LITE model over-estimates expression while blue indicates LITE model underestimates expression relative to NITE model.

Cell-level topic modeling also supported the pattern of primed accessibility preceding fate commitment. For example, the dynamics of genes ultimately expressed in the cortex whose accessibility was primed at the preceding branch point were described by cortex-specific expression topic e6 and branch-spanning accessibility topic a4 (Fig. 4d). As previously noted, accessibility topic a4 described a cell-wide change in chromatin state that did not correspond with a synchronous change in expression topic influence.

a

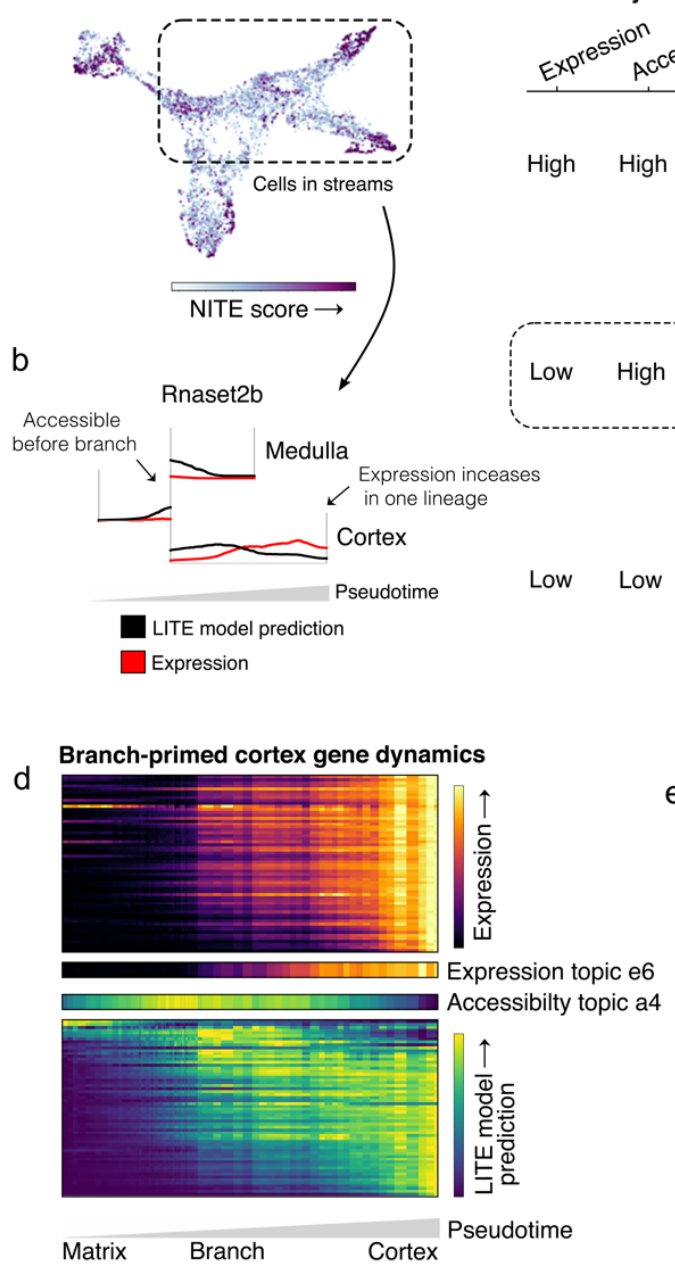

C

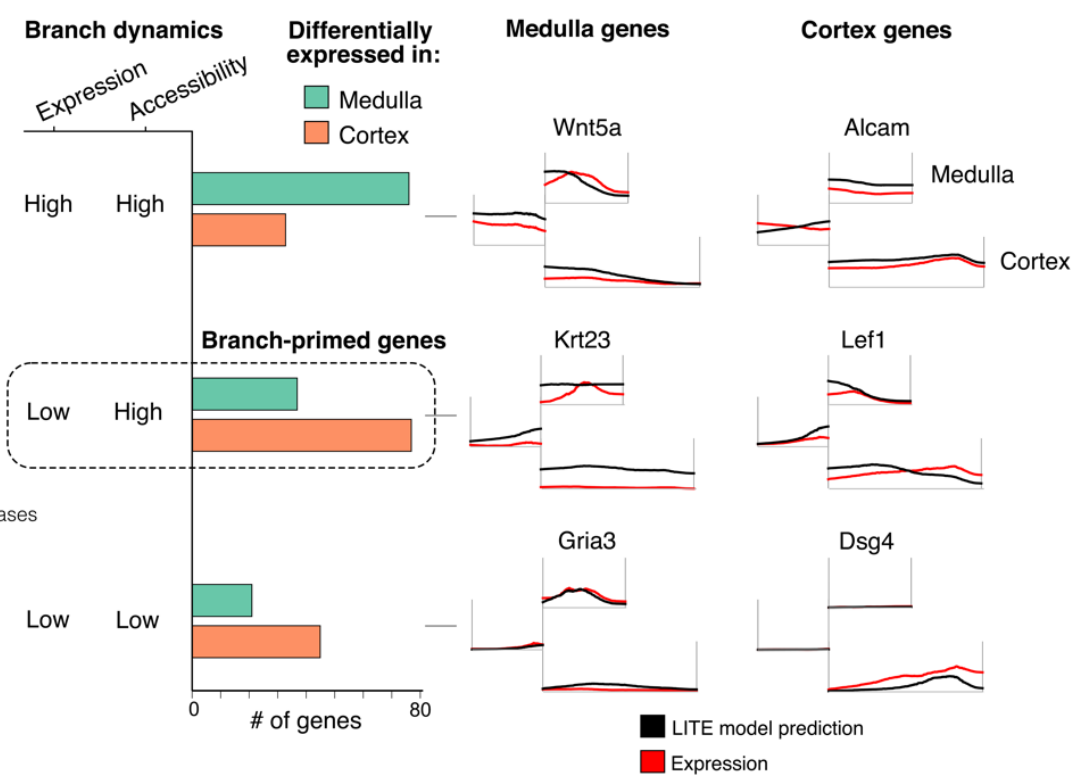

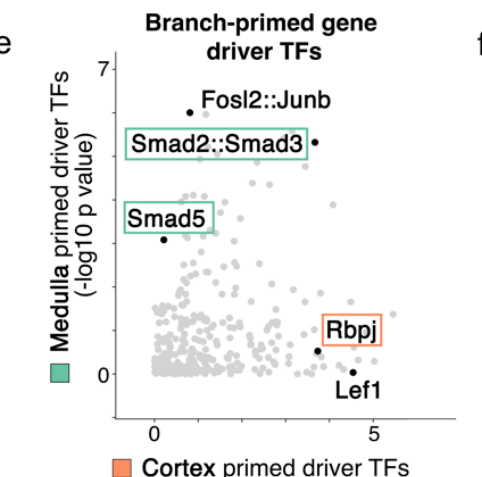

(- $\log 10 \mathrm{p}$ value)

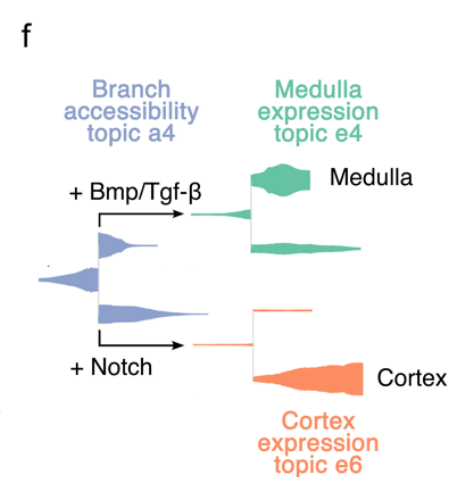

topic e6

Fig. 4 | Gene-level and cell-level analysis of NITE gene regulation in the hair follicle elucidated regulatory mechanisms of fate commitment. a, (top) NITE regulation score of each cell. Genome-wide expression in darker cells appeared to be more highly influenced by factors with minimal impact on local chromatin. $\mathbf{b}$, Stream graph of expression versus LITE model prediction of NITE gene Rnaset2b. c, (left) Regulatory classifications of medulla or cortex terminally expressed genes based on expression and local chromatin accessibility at the branch point between medulla and cortex lineages. Classifications colored by whether the genes were significantly upregulated in the medulla or cortex cells. (right) Example of each classification. Genes which were accessible at the branch but not yet highly expressed were denoted as branch-primed. $\mathbf{d}$, Interaction between gene-level regulation and cell-level topics. (top) Expression of branch-primed cortex genes increased after branch, correlating with expression topic e6. (bottom) 
LITE model prediction (local chromatin accessibility) of branch-primed genes increased before cortex commitment, correlating with accessibility topic a4. e, Driver transcription factor analysis of branch-primed medulla versus cortex genes. Branch-primed cortex genes showed regulation by Rbpj, effector of Notch signaling, while branch-primed medulla genes showed regulation by Smad5 and Smad2/3, effectors of Bmp and Tgf- $\beta$ signaling. f, Model for regulation of fate commitment in hair follicle depending on activation of distinct signaling pathways. Accessibility topic a4 opens chromatin around branch-primed genes at branch point between lineages. Depending on signal, branchprimed lineage-specific genes are expressed, enforcing lineage commitment.

Branch-primed genes that were subsequently conditionally expressed in medulla or cortex appeared to respond to a regulator of medulla or cortex fate commitment. MIRA pISD implicated Notch effector Rbpj as a top regulator of branch-primed cortex genes and Bmp/Tgf- $\beta$ induced Smad5/Smad2/3 as regulators of branch-primed medulla genes (Fig. 4e, Extended Data Fig. 5f), consistent with expression of genes associated with these factors' induction. (Extended Data Fig. 4b). Thus, MIRA determined that cells at the branch point have a chromatin state permissible to multiple fates, described by transitory accessibility topic a4, ultimately driven to medulla or cortex through the subsequent addition of a fate-defining signal, namely Bmp/Tgf- $\beta$ or Notch ${ }^{22,27,28}$ (Fig. 4f).

Overall, leveraging MIRA to systematically contrast expression and accessibility at single cell and locus resolution in the hair follicle revealed the fate commitment mechanism regulating the medulla and cortex lineages.

\section{MIRA captured two distinct spatiotemporal axes of differentiation in the interfollicular epidermis}

We next applied MIRA to a separate system in the same dataset ${ }^{3}$, the interfollicular epidermis (IFE). Two spatial axes of differentiation specify the IFE, one controlling the differentiation of basal stem cells into increasingly superficial epidermal layers (epidermal stratification axis) and another controlling basal cell invagination and follicular formation (follicular axis) ${ }^{29}$ (Fig. 5a). The latent structure of MIRA's joint topic representation again mimicked the spatial layout of this differentiation system, reconstructing the two axes of differentiation (Fig. 5b, Extended Data Fig. 6-7).

Furthermore, unlike prior reported analysis of this dataset ${ }^{3}$ that did not jointly model expression and accessibility, MIRA identified two distinct basal-spinous-granular trajectories. One trajectory, labeled "intermediate", was more transcriptionally and epigenetically similar to upper hair follicle structures, suggesting these cells were spatially proximal to the hair follicle and subject to more pro-follicular regulation. These "intermediate" basal cells showed activation of Egr2 expression and motifs, previously implicated in epidermal proliferation and wound healing ${ }^{30}$ (Extended Data Fig. 8a-b). By contrast, basal cells distant from the hair follicle showed stronger expression of Thbs1, consistent with prior work ${ }^{29}$ that identified two distinct populations of basal cells with Thbs 1 marking those distant from the hair follicle. Each of these two distinct basal cell niches produced their own columns of epidermal strata, which was captured by MIRA joint topic modeling.

Notably, the UMAP projection based only on expression obfuscated these distinct trajectories (Fig. 5c). RNA features were sufficient to distinguish the multi-stage transitions governing each basal-spinous-granular transformation but could not detect the lineage histories of each population. The accessibility-only representation, however, successfully aligned cells along lineages according to their distinct epigenetic characteristics (Fig. 5d). Projected together, 
bioRxiv preprint doi: https://doi.org/10.1101/2021.12.06.471401; this version posted December 7, 2021. The copyright holder for this preprint (which was not certified by peer review) is the author/funder, who has granted bioRxiv a license to display the preprint in perpetuity. It is made available under aCC-BY-NC-ND 4.0 International license.

the joint representation preserved the structure of the accessibility mode while integrating information of shared transcriptional identity from expression topics (Fig. 5b). In particular, expression topic e13 established cells with granular identities and captured co-upregulation of hallmark genes ${ }^{29}$ marking epidermal terminal differentiation (Fig. 5e-f).

a

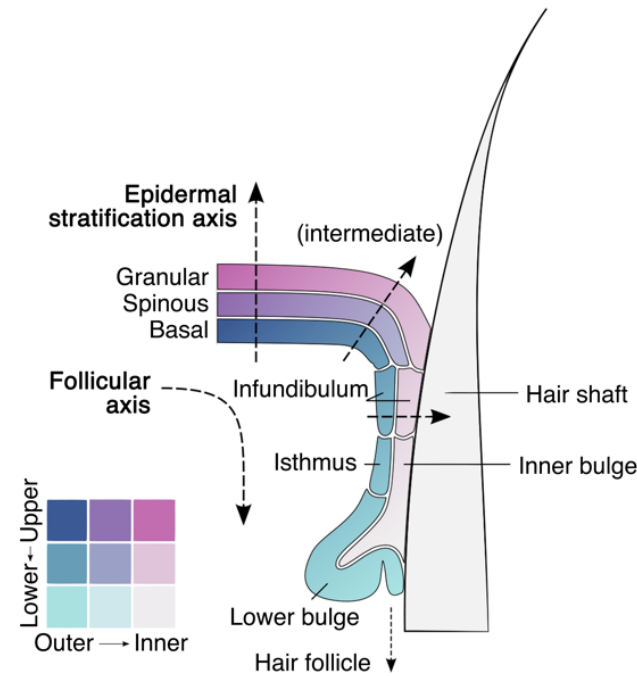

C

Expression
topic UMAP

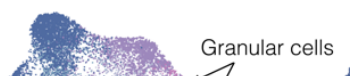

Accessibility
topic UMAP

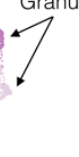

k.

g

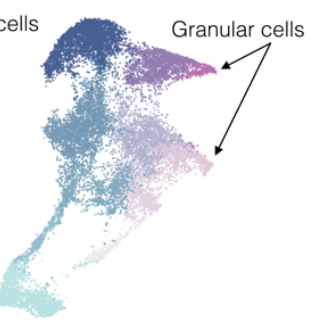

h b Joint expression and

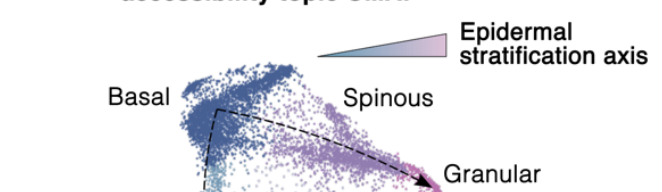

Intermediate

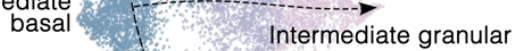

Intermediate spinous

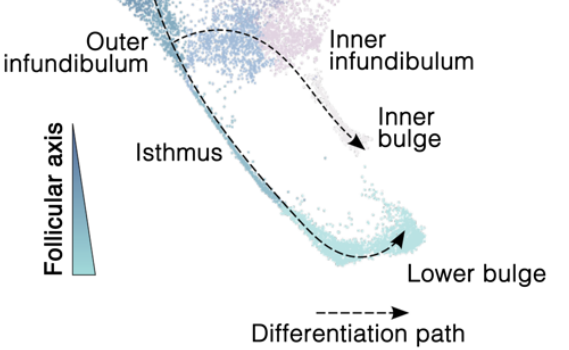

e Intermediate granular expression topic on joint UMAP

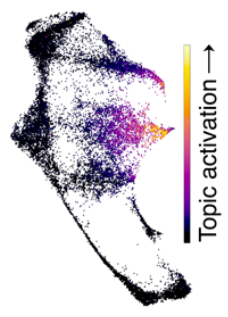

f

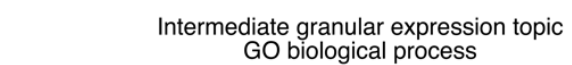
$\mathrm{GO}$ biological process

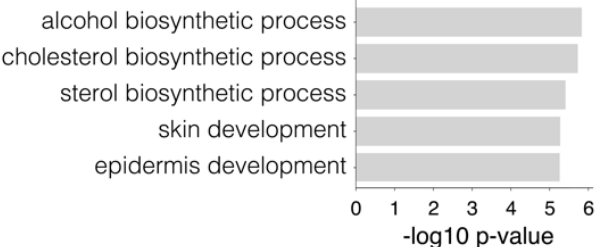

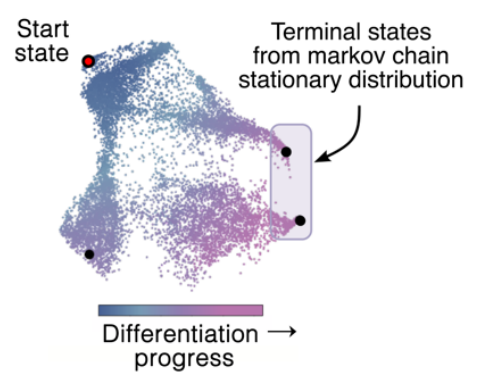

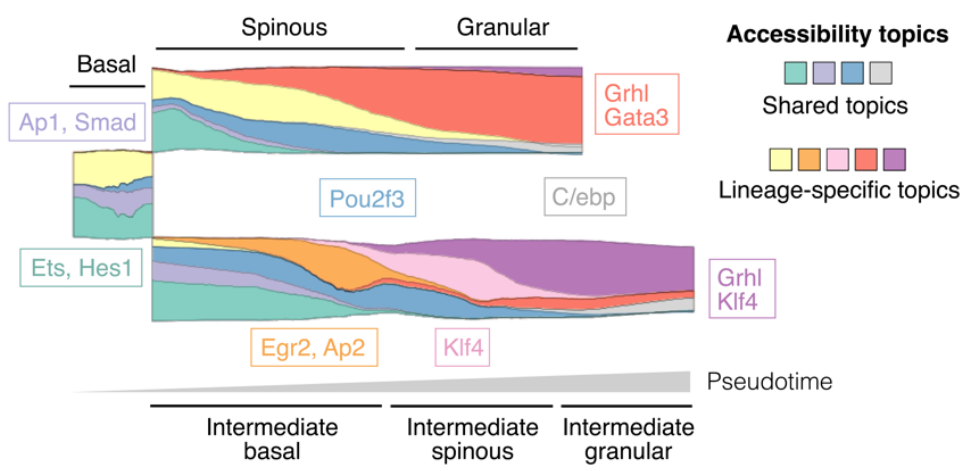

Fig. 5 | MIRA joint representation reconstructed complex multi-axis differentiation in the IFE. a, Anatomical model of mouse keratinocyte differentiation along epidermal and follicular axes. b, UMAP calculated from MIRA joint expression and accessibility topic representation. Dotted lines show constructed lineage structure resulting from two axes of differentiation. c, UMAP calculated from MIRA expression topics alone. d, UMAP calculated from MIRA accessibility topics alone. e, Activation of intermediate granular expression topic e8 on joint representation UMAP. f, Gene Ontology (GO) enrichment of top genes from intermediate granular expression topic e8. g, Two separate terminal states were identified from Markov chain model of differentiation starting from basal cells labeled "Start 
state". h, Stream graph of accessibility topic compositions of basal-spinous-granular (top) and intermediate basalspinous-granular (bottom) lineages. Top enriched factors shown in boxes with color indicating source topics.

The lineage tree inferred from the joint topic representation revealed both the shared and lineage-specific regulators shaping the spatial programs of the two basal-spinous-granular trajectories (Fig. 5g-h, Extended Data Table 3-4). Visualizing state changes through accessibility topics identified the shared regulatory influence of Hes on basal cells, followed by Pou2f3 on spinous cells and terminating in Grhl and C/ebp ${ }^{23,31}$ on granular cells in both trajectories. By contrast, lineage-specific accessibility topics distinguished the influence of Klf4 motifs $^{23}$ in "intermediate" spinous and granular cells, as opposed to Gata3 influence ${ }^{32}$ in granular cells arising from Thbs1+ basal cells more distant from the hair follicle.

We observed that expression in terminal populations was significantly enriched for NITE regulation, especially in terminal genes differentially-expressed between lineages $(p<0.05$, Wilcoxon) (Extended Data Fig. 8c-f). Again, terminal fate chromatin accessibility appeared to specify the available cell states, while transcription ultimately depended on additional spatial or signaling queues. Overall, MIRA elucidated the shared and lineage-specific mechanisms of differentiation along two parallel trajectories with distinct spatial regulation within the IFE.

\section{MIRA elucidated regulators driving key fate decisions in embryonic brain development} We next applied MIRA to an embryonic brain dataset (assayed on a different platform, 10x Multiome $)^{14}$ to determine the key factors driving the decision between inhibitory and excitatory neuronal fates, the balance of which is critical for normal brain development. In contrast to separate analyses of expression and accessibility, MIRA joint topic modeling constructed a high fidelity lineage tree demonstrating continuous flow from common progenitors to either astrocytes or neuronal progenitors that subsequently branched into inhibitory or excitatory neurons (Fig. 6a-b, Extended Data Fig. 9).

MIRA's high fidelity lineage tree was crucial to precisely contrasting the dynamics of expression and accessibility across the developmental continuum (Fig. 6c, Extended Data Fig. 10-13). The major expression topic e3 defining commitment to neuronal progenitors rather than astrocytes was enriched for cell cycle genes, likely reflecting the expansion of neuronal populations prior to commitment to the inhibitory or excitatory fate (Extended Data Fig. 12, Table 5). Subsequent inactivation of cell cycle topic e3 aligned with activation of accessibility topic a2, which was enriched for motifs of Ascl1, a pioneering transcription factor in neural progenitors known to promote cell cycle exit and differentiation ${ }^{33}$ (Fig. 6c; Extended Data Fig. 12, Table 6). Ascl1 motifs were also enriched in the early inhibitory accessibility topic a4, consistent with Ascl1's key role in inhibitory neuron differentiation ${ }^{34}$ (Fig. 6c-d, Extended Data Table 6).

In the alternative trajectory towards the excitatory fate, early excitatory accessibility topic a6 demonstrated depletion of Ascl1 motifs coordinated with increased Neurod1 motifs (Fig. 6ce, Extended Data Table 6). Ascl1 and Neurod1 belong to separate subgroups of basic helixloop-helix transcription factors ${ }^{35}$; and Neurod1 promotes differentiation into excitatory neurons, while Ascl1 specifies inhibitory neurons ${ }^{34}$.

MIRA topics contrasted the temporal progression of specification initiated by inhibitorydriving Ascl1 or excitatory-driving Neurod1. The inhibitory trajectory activated inhibitory maturation-driving Bdnf signaling ${ }^{36}$, culminating in the activation of GABA synapse components 
bioRxiv preprint doi: https://doi org/10.1101/2021.12 06 471401· this version posted December 7 2021. The copyright holder for this preprint (which was not certified by peer review) is the author/funder, who has granted bioRxiv a license to display the preprint in perpetuity. It is made available under aCC-BY-NC-ND 4.0 International license.

that define the terminal inhibitory fate (topic e13) (Fig. 6c; Extended Data Fig. 12, Table 5-6). Consistently, aligned terminal inhibitory accessibility topic a10 was enriched for Egr1 motifs, a downstream Bdnf effector that directly activates GABAergic neurotransmission genes ${ }^{37}$.

a

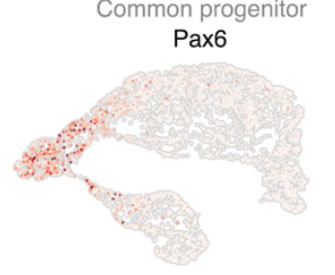

b

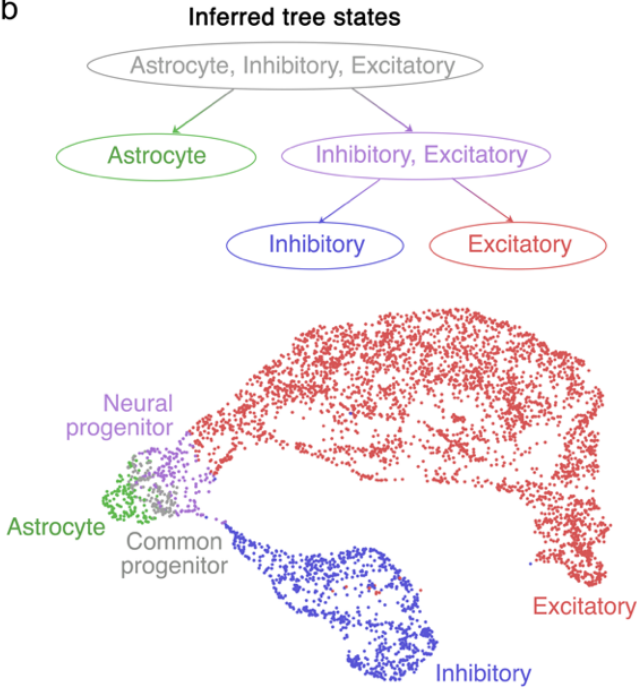

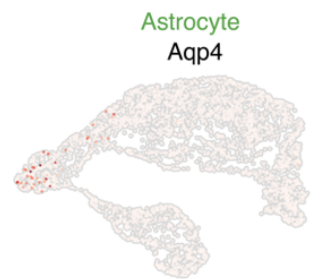

C
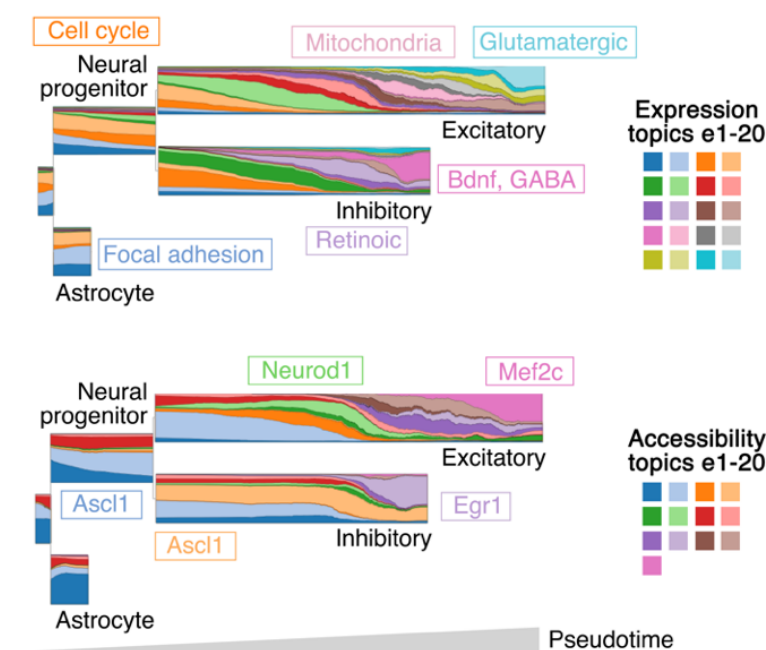

e

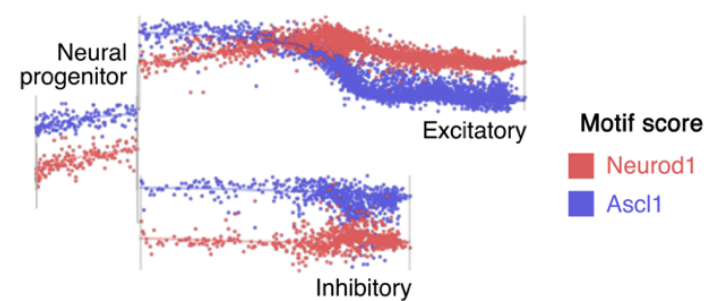

g

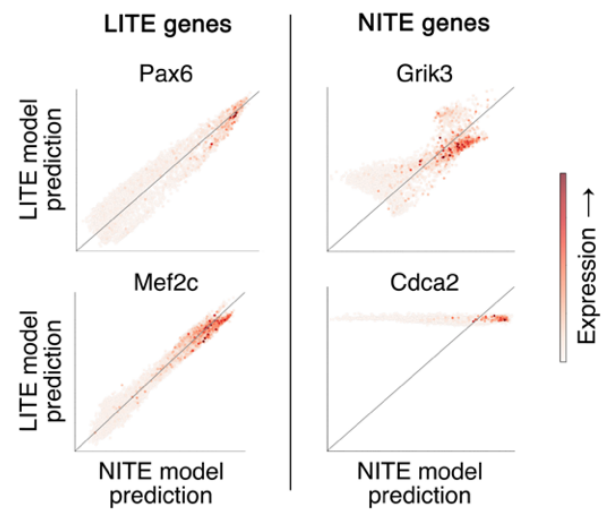

Fig. 6 | MIRA elucidated regulatory factors driving fate decisions at key developmental branch points in embryonic brain development. a, Expression of marker genes for common progenitor Pax6+ cells and terminal states of astrocytes, excitatory neurons, or inhibitory neurons. b, MIRA-inferred lineage tree and joint representation 
UMAP colored by inferred lineages. c, Stream graphs of expression and accessibility topic activation across pseudolineage trajectory. Pathways activated in expression topics and motifs enriched in accessibility topics are indicated by topic color. $\mathbf{d}$, Activation of early inhibitory and early excitatory accessibility topics on joint representation UMAP. e, Activation level of Ascl1 versus Neurod1 motif scores in each single cell along pseudolineage trajectory. $\mathbf{f}$, GO terms enriched in top 500 genes with NITE regulation where local chromatin accessibility state is insufficient to predict expression. g, Correlation of LITE versus NITE model predictions of expression of example genes with LITE versus NITE regulation.

The diverging excitatory branch first activated mitochondrial components important for supporting neuronal metabolic demands (topic e14) followed by terminal activation of glutamatergic synapse machinery, including glutamate transporters which uniquely distinguish excitatory neurons (topic e20) (Fig. 6c; Extended Data Fig. 13a, Table 5). Aligned terminal excitatory accessibility topic a13 was enriched for Mef2 motifs attributable to Mef2c given its expression in the excitatory branch, consistent with its known role in maintaining the excitatory/inhibitory balance by promoting excitatory differentiation ${ }^{38}$ (Fig. 6c; Extended Data Fig. 13b, Table 6).

In summary, contrasting expression and accessibility topics on MIRA's high fidelity joint lineage tree revealed the key regulators driving cell fate decisions at the neuronal branch point and exposed the subsequent temporal progression of specification into inhibitory or excitatory neuronal fates.

\section{MIRA revealed LITE and NITE genes in the embryonic brain}

To determine LITE and NITE genes in the embryonic brain, we trained MIRA RP models for the genes defining each expression and accessibility topic (Fig. 6f-g). Notable LITE genes included fate-driving transcription factors with tight spatiotemporal regulation such as common progenitor gene Pax6 and excitatory-promoting Mef2c. Conversely, NITE genes were enriched for cell cycle machinery as well as neuronal differentiation gene batteries composed of neurotransmitter and ion channel genes. Local chromatin landscape has been previously reported to have limited contribution to the activation of cell cycle genes ${ }^{3}$, consistent with NITE regulation. This may reflect a requirement for titration of genes governing each cell cycle stage that would be incompatible with the time needed to remodel the local chromatin landscape. Similarly, synaptic maintenance and plasticity may require fast-response regulation of neurotransmitter and ion channel genes, reflected as NITE regulation.

Analogously to the hair follicle and IFE, expression topics describing the neuronal progenitors were significantly enriched for LITE regulation, whereas after commitment to the excitatory or inhibitory fate, topics were significantly enriched for NITE regulation $(p<0.05$, Wilcoxon) (Extended Data Fig. 13c). Neuronal progenitor and early inhibitory regulator Ascl1 is known to be a pioneering transcription factor that remodels the chromatin landscape to regulate its targets ${ }^{33,39}$. By contrast, terminal inhibitory regulator Egr1 was previously reported to have non-pioneer-like properties ${ }^{40}$. Notably, targets predicted by MIRA pISD to be downstream of Ascl1 demonstrated significantly stronger LITE regulation than predicted Egr1 targets, potentially reflective of local chromatin remodeling by pioneering Ascl1 driving their expression $(p<0.05$, Wilcoxon) (Extended Data Fig. 13d).

\section{Discussion}

In sum, MIRA leverages cell-level topic modeling and gene-level RP modeling to rigorously 
contrast the spatiotemporal dynamics of single cell transcription versus chromatin accessibility to reveal how these mechanisms interact to orchestrate key fate decisions in developmental trajectories. In each system we analyzed, MIRA demonstrated the power of joint topic modeling of expression and accessibility data to infer high fidelity lineage trees that accurately captured complex spatiotemporal axes of differentiation. Mapping expression and accessibility topics onto the joint lineage tree illuminated the key regulators driving fate decisions at pivotal lineage branch points.

MIRA contrasted the dynamics of transcription and local chromatin accessibility to define the chromatin differential at each gene locus, revealing discrete gene modules regulated by primarily LITE or NITE mechanisms. Intriguingly, in all three systems that we tested from the skin $^{3}$ and brain ${ }^{14}$ datasets, earlier-expressed genes were enriched for LITE regulation. LITE regulation of earlier-expressed genes may reflect the importance of strict regulation requiring extensive chromatin remodeling for their expression followed by strong silencing in fates where their aberrant expression would have devastating consequences. Conversely, gene batteries important for maintaining terminal cell function were less reliant on local chromatin remodeling for their regulation, suggesting larger influence by mechanisms such as cell signaling that allow titration of transcription to fulfill fluctuating cell needs.

Among NITE-regulated genes, we also noted genes with primed accessibility at lineage branch points that showed subsequent lineage-specific activation in response to a fate-defining force such as signaling, presumably via binding or activation of a factor with minimal impact on local accessibility. In these cases, accessibility appeared to reflect a plastic cell identity encoding the available transcriptional states of the cell where ultimate transcription ensued in response to the cell's spatial or signaling niche. Future work is warranted to further determine the logic of when cells employ LITE versus NITE mechanisms to regulate distinct cellular processes.

In conclusion, MIRA leverages principled probabilistic cell-level topic modeling and gene-level RP modeling to precisely contrast the spatiotemporal dynamics of transcription and local chromatin accessibility at unprecedented resolution. MIRA thereby exposes the key regulators driving fate decisions at lineage branch points and reveals the distinct circuitry regulating fate commitment versus terminal identity. MIRA thus represents a much-needed computational tool for deeply integrated analysis of the rapidly expanding wealth of multimodal data in the single cell field. Moving beyond visualization, MIRA enables rigorous interrogation of the transcriptional and epigenetic mechanisms interacting to drive dynamic biological systems.

\section{Methods}

Complete methods available in Extended Data.

\section{Acknowledgements}

We thank the X. Shirley Liu Lab members, Matthew Oser, and Kai Wucherpfennig for helpful scientific discussions. This work was supported by the National Institutes of Health (NIH) grant U24 CA237617 to CAM. CVT was supported by NIH T32GM007748.

\section{Author information}

AWL developed MIRA, designed analyses, and analyzed the SHARE-seq dataset. CVT co- 
developed MIRA, designed analyses, and analyzed the 10x dataset. HL and MB contributed to analysis design. XSL and CAM designed analyses and supervised the work. AWL, CVT, XSL, and CAM wrote the manuscript. AWL and CAM originated the work. All authors edited and approved the manuscript.

Competing interests: $\mathrm{MB}$ is a consultant to and receives sponsored research support from Novartis. MB serves on the SAB of H3 Biomedicine, Kronos Bio, and GV20 Oncotherapy. XSL is a cofounder, board member, SAB member, and consultant of GV20 Oncotherapy and its subsidiaries; is a stockholder of BMY, TMO, WBA, ABT, ABBV, and JNJ; and received research funding from Takeda, Sanofi, and Novartis.

\section{Code availability}

MIRA is available as a Python package at https://github.com/cistrome/MIRA.

\section{References}

1. Chen, S., Lake, B. B. \& Zhang, K. High-throughput sequencing of the transcriptome and chromatin accessibility in the same cell. Nat. Biotechnol. 37, 1452-1457 (2019).

2. Cao, J. et al. Joint profiling of chromatin accessibility and gene expression in thousands of single cells. Science 361, 1380-1385 (2018).

3. Ma, S. et al. Chromatin Potential Identified by Shared Single-Cell Profiling of RNA and Chromatin. Cell 183, 1103-1116.e20 (2020).

4. Zhu, C. et al. An ultra high-throughput method for single-cell joint analysis of open chromatin and transcriptome. Nat. Struct. Mol. Biol. 26, 1063-1070 (2019).

5. Duren, Z., Chen, X., Xin, J., Wang, Y. \& Wong, W. H. Time course regulatory analysis based on paired expression and chromatin accessibility data. Genome Res. 30, 622-634 (2020).

6. Gayoso, A. et al. scvi-tools: a library for deep probabilistic analysis of single-cell omics data. bioRxiv 2021.04.28.441833 (2021) doi:10.1101/2021.04.28.441833.

7. Gong, B., Zhou, Y. \& Purdom, E. Cobolt: Joint analysis of multimodal single-cell sequencing data. bioRxiv (2021).

8. Minoura, K., Abe, K., Nam, H., Nishikawa, H. \& Shimamura, T. scMM: Mixture-of-experts multimodal deep generative model for single-cell multiomics data analysis. bioRxiv 2021.02.18.431907 (2021) doi:10.1101/2021.02.18.431907.

9. Chen, H., Ryu, J., Vinyard, M., Lerer, A. \& Pinello, L. SIMBA: SIngle-cell eMBedding Along with features. bioRxiv 2021.10.17.464750 (2021) doi:10.1101/2021.10.17.464750.

10. Lin, Y. et al. scJoint: transfer learning for data integration of single-cell RNA-seq and ATACseq. bioRxiv 2020.12.31.424916 (2021) doi:10.1101/2020.12.31.424916.

11. Duren, Z. et al. Integrative analysis of single-cell genomics data by coupled nonnegative matrix factorizations. Proc. Natl. Acad. Sci. U. S. A. 115, 7723-7728 (2018).

12. Lara-Astiaso, D. et al. Chromatin state dynamics during blood formation. Science (2014) doi:10.1126/science.1256271.

13. Rada-Iglesias, A. et al. A unique chromatin signature uncovers early developmental enhancers in humans. Nature 470, 279-283 (2011).

14. Datasets - 10x Genomics. https://support.10xgenomics.com/single-cell-multiome-atacgex/datasets.

15. Blei, D. M. Probabilistic topic models. Commun. ACM 55, 77-84 (2012).

16. Zhao, Y., Cai, H., Zhang, Z., Tang, J. \& Li, Y. Learning interpretable cellular and gene signature embeddings from single-cell transcriptomic data. Nat. Commun. 12, 5261 (2021). 
17. Bravo González-Blas, C. et al. cisTopic: cis-regulatory topic modeling on single-cell ATACseq data. Nat. Methods 16, 397-400 (2019).

18. Kingma, D. P. \& Welling, M. Auto-Encoding Variational Bayes. arXiv [stat.ML] (2013).

19. Blei, D. M. Latent Dirichlet Allocation. J. Mach. Learn. Res. 3, 993-1022 (2003).

20. Wang, S. et al. Target analysis by integration of transcriptome and ChIP-seq data with BETA. Nat. Protoc. 8, 2502-2515 (2013).

21. Qin, Q. et al. Lisa: inferring transcriptional regulators through integrative modeling of public chromatin accessibility and ChIP-seq data. Genome Biol. 21, 32 (2020).

22. Schneider, M. R., Schmidt-Ullrich, R. \& Paus, R. The hair follicle as a dynamic miniorgan. Curr. Biol. 19, R132-42 (2009).

23. Blanpain, C. \& Fuchs, E. Epidermal homeostasis: a balancing act of stem cells in the skin. Nat. Rev. Mol. Cell Biol. 10, 207-217 (2009).

24. L. Byron \& M. Wattenberg. Stacked Graphs - Geometry \& Aesthetics. IEEE Trans. Vis. Comput. Graph. 14, 1245-1252 (2008).

25. Soma, T., Ogo, M., Suzuki, J., Takahashi, T. \& Hibino, T. Analysis of Apoptotic Cell Death in Human Hair Follicles In Vivo andln Vitro. J. Invest. Dermatol. 111, 948-954 (1998).

26. Cui, C.-Y. et al. Ectodysplasin regulates the lymphotoxin-beta pathway for hair differentiation. Proc. Natl. Acad. Sci. U. S. A. 103, 9142-9147 (2006).

27. Pan, Y. et al. gamma-secretase functions through Notch signaling to maintain skin appendages but is not required for their patterning or initial morphogenesis. Dev. Cell 7, 731-743 (2004).

28. Genander, M. et al. BMP signaling and its PSMAD1/5 target genes differentially regulate hair follicle stem cell lineages. Cell Stem Cell 15, 619-633 (2014).

29. Joost, S. et al. Single-Cell Transcriptomics Reveals that Differentiation and Spatial Signatures Shape Epidermal and Hair Follicle Heterogeneity. Cell Syst 3, 221-237.e9 (2016).

30. Grose, R., Harris, B. S., Cooper, L., Topilko, P. \& Martin, P. Immediate early genes krox-24 and krox-20 are rapidly up-regulated after wounding in the embryonic and adult mouse. Dev. Dyn. 223, 371-378 (2002).

31. Hildesheim, J. et al. The hSkn-1a POU transcription factor enhances epidermal stratification by promoting keratinocyte proliferation. J. Cell Sci. 114, 1913-1923 (2001).

32. Zeitvogel, J. et al. GATA3 regulates FLG and FLG2 expression in human primary keratinocytes. Sci. Rep. 7, 1-11 (2017).

33. Raposo, A. A. S. F. et al. Ascl1 Coordinately Regulates Gene Expression and the Chromatin Landscape during Neurogenesis. Cell Rep. 10, 1544-1556 (2015).

34. Yang, N. et al. Generation of pure GABAergic neurons by transcription factor programming. Nat. Methods 14, 621-628 (2017).

35. de Martin, X., Sodaei, R. \& Santpere, G. Mechanisms of Binding Specificity among bHLH Transcription Factors. Int. J. Mol. Sci. 22, (2021).

36. Porcher, C., Medina, I. \& Gaiarsa, J.-L. Mechanism of BDNF Modulation in GABAergic Synaptic Transmission in Healthy and Disease Brains. Front. Cell. Neurosci. 12, 273 (2018).

37. Mo, J. et al. Early growth response 1 (Egr-1) directly regulates GABAA receptor $\alpha 2$, $\alpha 4$, and $\theta$ subunits in the hippocampus. J. Neurochem. 133, 489-500 (2015).

38. Harrington, A. J. et al. MEF2C regulates cortical inhibitory and excitatory synapses and behaviors relevant to neurodevelopmental disorders. Elife 5, (2016).

39. Park, N. I. et al. ASCL1 Reorganizes Chromatin to Direct Neuronal Fate and Suppress Tumorigenicity of Glioblastoma Stem Cells. Cell Stem Cell 21, 411 (2017).

40. Chen, C.-H. et al. Determinants of transcription factor regulatory range. Nat. Commun. 11, $2472(2020)$. 